\title{
The Effect of Personality on Online Game Flow Experience and the Eye Blink Rate as an Objective Indicator
}

\author{
Pei-Luen Patrick Rau, Yu Chien Tseng, Xiao Dong, Caihong Jiang, and Cuiling Chen \\ Institute of Human Factors \& Ergonomics, Department of Industrial Engineering, Tsinghua University, Beijing 100084, China \\ Correspondence should be addressed to Pei-Luen Patrick Rau; rpl@mail.tsinghua.edu.cn
}

Received 8 January 2017; Revised 6 March 2017; Accepted 20 April 2017; Published 18 June 2017

Academic Editor: Pietro Cipresso

Copyright (C) 2017 Pei-Luen Patrick Rau et al. This is an open access article distributed under the Creative Commons Attribution License, which permits unrestricted use, distribution, and reproduction in any medium, provided the original work is properly cited.

This study aimed to explore the effects of dominant and compliant personalities, on both flow experience and the external characteristics of flow experience. A total of 48 participants were recruited to play an online game and subsequently asked to recall the songs they had heard while they were playing the game. Eye blink rate was recorded. The results demonstrated that (1) the participant was immersed in the game more if he/she was relatively dominant or noncompliant; (2) the perceptions about the external environment declined remarkably while being in a flow state; and (3) eye blink rates decreased only when the flow happened at the beginning of the game, rather than throughout the whole process. The results suggested that gamers who tend to be dominant or noncompliant were more likely to experience flow. Eye blink rate and perceptions of the external environment could be objective indicators of flow experience.

\section{Introduction}

With the advent of Web 2.0, user experiences have become a significant topic of interest for researchers. Flow experience, the optimal user experience, has drawn the attention of many researchers, especially those studying topics within the virtual world. Many factors may influence an individual's flow experience. Personalities can influence the way we perceive and think about the world. However, how an individual's personality influences our flow experience in online games is still not clear. This is the first question that the present study sought to explore. In addition, we attempted to clarify the process of judging whether or not a person is in a flow state. Also, what indicators, especially those that are objective enough for us to evaluate flow, could be used to determine whether someone is in a flow state.

\section{Literature Review}

2.1. Flow Experience. Csikszentmihalyi (1975) first used the theory of flow to determine that people often do not need any prizes or punishment in order to push them to participate in activities they are interested in doing [1]. This phenomenon is especially applicable to artists, regardless of whether the art is related to work or leisure. Artists concentrate solely on their work and are willing to complete it without any external rewards or additional monetary costs or time. Csikszentmihalyi referred to this as "flow." According to Csikszentmihalyi, flow tends to occur when one's skills are fully involved in overcoming a challenge that is considered manageable. Optimal experiences usually involve a fine balance between one's ability to act and other available opportunities. Otherwise, the flow state will come to an end. There are three elements that best describe flow: antecedent conditions, characteristics, and consequences of experience [2]. Antecedent conditions refer to the balance of challenge and skills, the clear definition of goals, and immediate feedback. Characteristics include action awareness merging, effortlessness, intense concentration on the task at hand, a sense of control, and enjoyment. Consequences of experience are the loss of self-consciousness, transformation of time, and autotelic experiences.

2.2. Dominance/Compliance Personality and Flow Experience. Two distinct personalities, dominance and conformity, are 
thought to exert influence on an individual's flow experience. Dominance refers to controlling one's human environment, to influence or direct the behavior of others through suggestion, seduction, persuasion, or command, and to dissuade, restrain, or prohibit [3-5]. Dominant people are usually risktakers, pugnacious, fearless, decisive, straight, innovative, dauntless, problem solvers, and self-inspired. They act as the leader in a group. These personal characteristics allow them to concentrate more on their own experiences and encounter more joy, which may indicate that people with high dominance may have a greater chance to experience flow compared to people with low dominance.

There is some empirical evidence to support that dominant people experience flow more easily, compared with nondominant people. Csikszentmihalyi and LeFevre (1989) have found that managers are more likely to experience flow at work, compared with blue-collar workers [6]. Managers are in a dominant position at work and demonstrate dominance through their leadership, while blue-collar workers passively accept tasks or directions from their supervisors or managers, because they are in a nondominant position [7]. Han and Yoo (2014) examined the effects of an autotelic personality on flow experience in cyclists. Their study found that people with an autotelic personality, which consisted of curiosity, persistence, altruism, independence, and vitality personality factors, could significantly influence levels of flow experience [8]. Heller et al. (2015) investigated the correlations between personality traits with Eysenck's Personality Profiler and flow experience by the Practice Flow Inventory, in 120 amateur vocal students. The results revealed that participants with high extraversion-scores experienced significantly more flow than less extraverted persons. Lesser flow experience levels were related to high neuroticism-scores. Heller et al. also found that the certainty in handling an instrument for singers is essential to arouse a feeling of flow [9], indicating that the feeling of control is vital for people to obtain flow. People with a dominant personality tend to control their environment and own a high degree of control $[4,5]$. It was also reported that people with a strong internal control experienced more flow than people with weak internal control [10]. Based on the results referred to above, it is hypothesized that dominant people might experience more flow than nondominant people.

Myers (1987) defines conformity to be "a change in behavior or belief as a result of real or imagined group pressure." Myers stated that sometimes we conform without really believing in what we are doing and that this insincere outward conformity is called compliance. Other times we genuinely believe in what the group has convinced us to do. This sincere inward conformity is called acceptance. Compliance is the process of changing one's own idea passively, under the pressure of a group or society [11, 12]. When a person can be easily led or influenced by external factors, he or she may find it hard to concentrate on the task. But, one of the essential conditions required to experience flow is to fully focus on the task at hand. Thus, people with high compliance may be less likely to experience flow compared to people with low compliance. The empirical evidence for supporting this view is scarce. Ross and Keiser (2014) examined the effect of personality, using the Five Factor Model of personality, and the flow propensity with the Dispositional Flow Scale 2 (DFS-2), in 316 young adults. They found that neuroticism, extraversion, agreeableness, and conscientiousness predicted global flow propensity, which accounted for $38 \%$ of the measured variance. Among these characteristics, extraversion (with dominance as a subdimension), positively predicted an individual's global flow propensity. But agreeableness (with compliance as a subdimension) negatively predicted an individual's global flow propensity [13]. These results may indicate that compliant people are negatively correlated with flow; that is, the more compliant characteristics that people have, the less flow they are likely to experience. Thus, we inferred that compliant people were less likely to experience flow than noncompliant people.

2.3. The External Expression of Flow Experience. People experiencing flow might display different behaviors compared to those not in a flow state. One important change is the perception of the external environment, while another factor is eye blink rate.

Perceptions of the External Environment. People in a flow state often focus their concentration on what he or she is doing, with almost all of their attention and resources absorbed by the current activity [6, 14]. A loss of self-awareness [15] may lead the external information to be poorly perceived. Perception of one's external environment begins with the activation of sensory receptors, with the information stored in a memory system. This process is referred to as the "sensory register," which is designed to store a record of the information received by receptor cells [16]. The sensory register is an important tool, as processing sensory information to obtain a meaning and adding information to the sensory pattern is not accomplished instantaneously. Yet, the ability of the brain to process multiple patterns of sensory information is limited. Thus, only part of the information can be selected by the working memory, to further enter into an individual's long-term memory. Most information is lost due to a lack of further processing. This process is called "attention filter theory." Flow theory also states that when a person goes into a flow status, brain activity usually focuses on the task, and thus the bandwidth of the information reception channel decreases [7]. Therefore, the amount of information from the external environment, which enters into an individual's working memory, is significantly reduced.

Eye Blink Rate. Currently, there is limited literature that has explored the adoption of eye tracking, as a means to evaluate computer games [17-19]. In these studies, eye fixation [20, 21], eye gaze [22], and saccades [23] are the key factors used to determine eye movement indicators. The eye blink rate, still called spontaneous eye blink in some studies, is also an important indicator in eye tracking studies. Some researchers have used eye blink rates as an indicator in some fields. Veltman and Gaillard (1998) reported that increases in visual information inhibited the eye blink, and in contrast, increasing the difficulty of a memory task led to an increase in the blink rate [24]. These results were consistent with a study 
by Acosta, Gallar, and Belmonte (1999) [25], who found that eye blink rates drop when people performed a task requiring strong visual attention. Wu, Begley, Zhang, Adebisi, and Simpson (2011) [26] confirmed these findings and reported that eye blink rates decreased with greater concentration. When investigating changes in the eye blink rates of children engaged in video games, researchers have demonstrated a decrease in blinking when the game was in progress [27]. Kim, Min, and Park (2013) also confirmed these findings. They found that the frequency of eye blink significantly decreased by $12 \%$, when game playing participants were in high flow level compared with when they were in a low flow level [28]. Based on the studies above, we anticipated that the eye blink rate reduced when people were immersed in computer games, in other words, when people experienced flow.

Hypothesis. Based on the discussions above, a subsequent research framework was proposed (see Figure 1). The present study included two independent variables: personality (dominant/nondominant, compliant/noncompliant) and a participant's game status (flow versus nonflow). The dependent variables were the extent of flow experience, perception of external environment, and blink rate.

Four hypotheses were formulated.

Hypothesis 1 (H1). Compared with being nondominant, dominant individuals are more likely to experience flow.

Hypothesis 2 (H2). Compared with being compliant, individuals are more likely to experience flow if he/she is relatively noncompliant.

Hypothesis 3 (H3). A gamer's perceptions of their external environment will decline remarkably when he/she is in a flow state.

Hypothesis 4 (H4). A gamer's blink rate will decrease if he/she is experiencing flow.

\section{Methodology}

3.1. Participants. Firstly, we conducted a recruitment process in the city of Beijing for volunteers to take part in our study. We used posters to promote the study, with 129 students prepared to take part. Secondly, all volunteers were screened based on the following two criteria: (1) having at least three years of experience in playing Warcraft 3 and (2) a relatively high (dominant score is higher than 49 or compliant score is higher than 45) or low (dominant score is lower than 31 or compliant score is lower than 35) personality scale score. Finally, a total of 48 male participants with four personality types (dominance, nondominance, compliance, and noncompliance) were selected to take part in the study. Each personality type had 12 participants. All participants were undergraduate or graduate students from one of several universities in Beijing. The participants had little or no prior knowledge about the proposed tasks. The participant's ages ranged from 19 to $26(\mathrm{M}=22, \mathrm{SD}=1.71)$ years. Participants

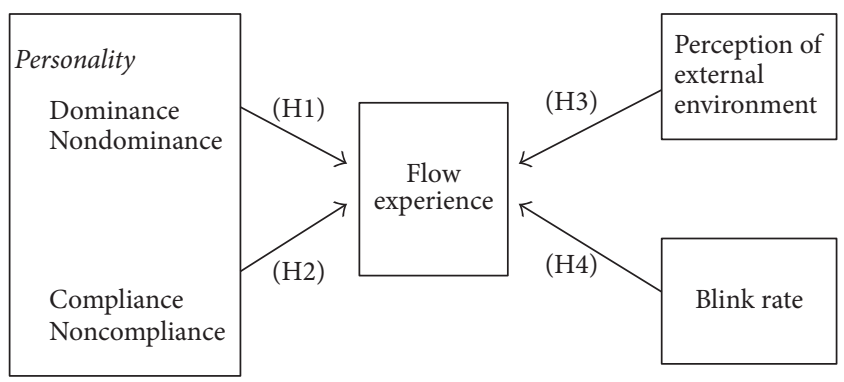

FIGURE 1: Research framework.

were allocated into four groups: dominant (range: 49-70) versus nondominant groups (range: 22-31) and compliant (range: 45-60) versus noncompliant (range: 20-35) groups, which were done according to the personality scale score.

Due to the limitation of the number of players, only 8 participants could play games simultaneously. As such, the 48 participants were assigned to six groups and each group consisted of four types of personalities (dominance, nondominance, compliance, and noncompliance) with two participants each. Each personality type had 12 participants. To preclude the effect of the degree of familiarity on the interaction frequency among them, each group of the participants $(N=8)$ were randomly selected from the 48 volunteers. The participants confirmed that they did not know each other. In addition, participants were asked to use their assigned code as their player name, to be sure that interactions with each other were objective.

\subsection{Tasks}

Task 1: Recording Eye Blink Films. All participants were twice asked to put on a head mounted device to record eye measurements. Both of the recordings were conducted in playing mode. The first eye recording was conducted for three minutes when participants were not in a flow experience status, and the second recording was conducted for 70 minutes when participants were in a flow state. After the experiment, the two films were loaded into the "Blink Statistic" program one by one. The blink rate of each film was counted separately.

Task 2: Playing Warcraft 3. The purpose of the game was to protect a basement. In the game, each of the participants chooses a character, and then control its actions with the objective to kill their enemies. Their accumulated money and experiences in the game increased with the number of enemies that they killed. Money could buy equipment, while experience could raise the player's abilities. A boss would appear at 60-70 minutes in games. Eight participants could communicate and cooperate with each other at any time in the game, unless their basement was breached and the game was over. If the game finished within 105 minutes, participants were asked to play another round until the time was up.

Task 3: Song Recognition Test. Participants were asked to answer ten questions without a time limitation. For each 

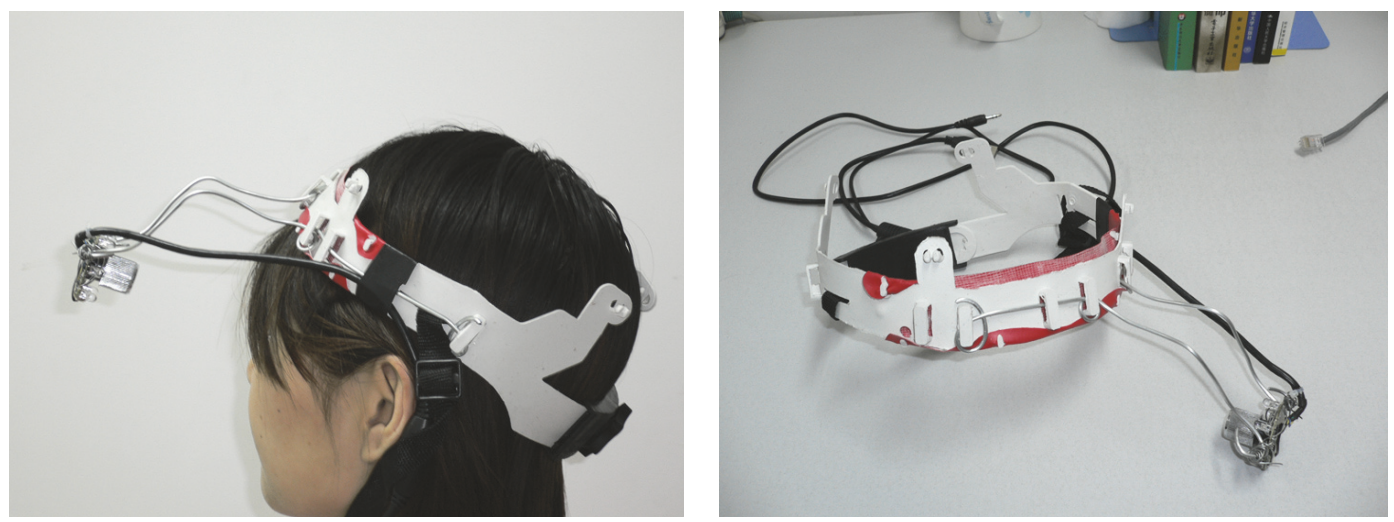

FIGURE 2: Head mounted device.

question, participants were required to listen to a song and recall whether they had heard it when they were playing Warcraft 3. They could adjust the scroll bar of the music player in order to listen to every part of the song. Once they had submitted an answer for each question, it could not be changed.

3.3. Experimental Design and Variables. Personality was considered a between-subject variable and consisted of dominant/nondominant or compliant/noncompliant. Game stage (idle or playing game) was a within-subject variable. The dependent variables were blink rate, degree of flow experience, and perception of the external environment.

Flow experience was measured using a questionnaire with 28 questions [18], which were rated on a scale of 1 (disagree strongly) to 7 (agree strongly). Cronbach's alpha of the original questionnaire was between 0.79 and 0.94 , implying a high level of reliability. Cronbach's alpha coefficient in this study was 0.92 , indicating an excellent reliability.

The "Blink Statistic" program measured eye blink rates. When analyzing the blink rates during a flow experience, the normal condition of their eye blinks, which was found on the first recorded film for each participant, was used. Perception of the external environment was measured using the number of correct answers in the song recognition assessment.

3.4. Apparatus and System. The study was conducted in a reserved room in a cybercafe. Each participant was equipped with two personal computers, one for the eye blink recording and the other for playing Warcraft 3.

Two Personal Computers. Two personal computers in the cybercafe were used for each participant. The detailed configuration of these computers were Pentium D 2.8 G CPU, 1 G RAM, Geforce Four 6600 display card, desktop monitor with a 17 -inch LCD monitor with a display resolution $1024 \times 768$, and Windows XP Professional operating system.

Head Mounted Device. This was a piece of equipment used to measure the condition of the eye within a continuous period. The device was made up of a webcam and headgear (see Figure 2). The headgear was removed from a safety helmet

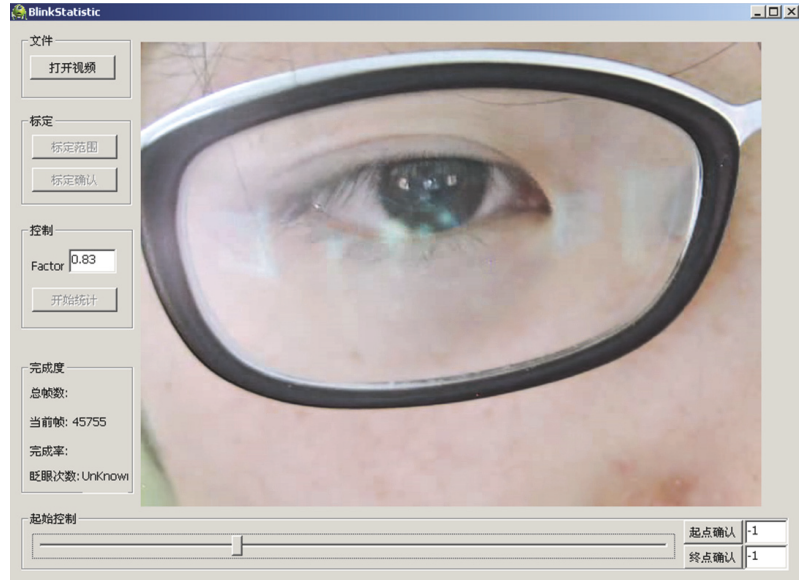

FIGURE 3: Interface of "blink statistic."

to keep the webcam stable on the head. The headgear was adjusted to suit the size of each of the participant's heads. The lens and chip were taken from a webcam with a resolution of five megapixels and plugged into a computer. At most, the webcam could process 30 frames, with a resolution of $640 * 480$ per second. The webcam was tied with wire, fixed on the headgear, and stretched out. During the recording process, the webcam was adjusted to a distance of $8 \mathrm{~cm}$ and a $20^{\circ}$ elevation degree to the front of the left eye.

Systems. The user interface of the experiment was designed as a webpage and developed using Macromedia Dreamweaver running on Windows XP SP2. The webpage would automatically save individual files for each subject. To avoid the effect of latency of the network transfer, this experiment was carried out without the use of Internet.

A self-written program called "Blink Statistic" was used for counting the number of eye blinks, as well as the blink rate over a long period of time (see Figure 3). The operating system platform for "Blink Statistic" was Microsoft Windows XP, DotNET Framework 1.1, and the display color quality was 32 bits. It was written in Microsoft Visual C++ language, developed by Microsoft Visual Studio.Net 2003. 


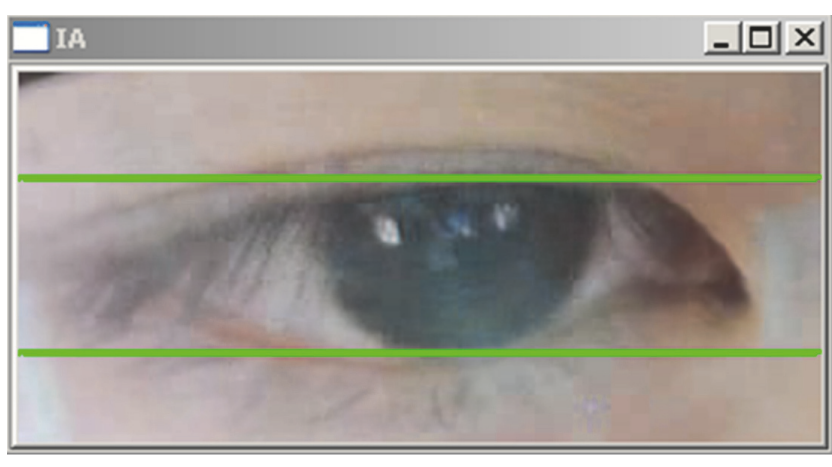

FIGURE 4: Demarcate the eye area.

After the experiment, all recorded films were loaded into "Blink Statistic." The researcher first needed to demarcate the spot of the eye manually, and then the system could scan the eyeballs in a limited area, ultimately deciding the position of the upper and lower eyelids (see Figure 4). Finally, the program automatically calculated and showed the total number of eye blinks and the blink rate of each film. The time taken to count the film takes only one-third of the original time span.

Game. Warcraft 3 was chosen for this experiment for the following reasons: (1) Internet games are highly self-centric and require a great deal of concentration for an individual to interact with virtual characters in a confined environment [29]; (2) it is a game conducted in real time with all players playing simultaneously, unlike card games that play in turn; and (3) the style of this game is rather simplistic and the participant's states can be easily controlled (players were all on the same side versus the computer). Participants could only choose one of the 21 characters, which were all at the same level at the beginning of the game. Finally, (4) this game takes advantage of the characteristics of online games, enabling participants to easily communicate. For example, a dialog box appears at the bottom left corner of the window after pressing "enter," and players can then communicate with each other by typing messages.

3.5. Procedure. The whole experimental study can be divided into three stages: before playing Warcraft 3; while playing Warcraft 3; and after playing Warcraft 3 (flow experience). Participants experienced all of these three stages. Finally, participants were asked to complete three questionnaires, the flow experience questionnaire, the satisfaction questionnaire, and a questionnaire regarding their self-evaluation of the games performance.

Before playing Warcraft 3 stage: an introduction to the study was provided to participants, informing them of how it would be conducted and what they would be required to do. All participants provided written informed consent and received financial compensation for their participation. The first eye recording was conducted during this stage. While playing Warcraft 3 stage: participants played the game during which time the eye recordings were performed. After playing Warcraft 3 stage: a song recognition test and the flow questionnaire were completed.

\section{Results}

4.1. The Effect of Personality on Flow Experience. The results showed that the dominant personality had an overwhelming impact on flow experience, $F(1,21)=6.94, p<.05$. The dominant participants $(n=12)$ had a higher flow experience score $(\mathrm{M}=131.58, \mathrm{SD}=24.49)$ than the nondominant group $(n=12 ; \mathrm{M}=100.42, \mathrm{SD}=25.32)$. These findings support $(\mathrm{H1})$. The compliant personality type also had a significant effect on flow experience, $F(1,21)=4.51, p<.05$. The compliant group $(n=12)$ had a lower flow experience score $(M=108.17$, $\mathrm{SD}=22.98)$ compared with the noncompliant group $(n=12$; $\mathrm{M}=124.33, \mathrm{SD}=12.91)$. Hypothesis 2 was also supported.

\subsection{The Effect of Different Flow States on Gamer's Perception of} External Environment. In order to explore the effect of flow states on a gamer's perception of the external environment and blink rates, all the participant's flow scores $(n=48)$ were ranked in an ascending order. Twenty-five percent of the highest scores in the flow questionnaire were defined as the flow group (scores higher than 134; $n=12$ ), and twenty-five percent of the lowest scores in the flow questionnaire were defined as the nonflow group (score lower than $98 ; n=12$ ).

An independent $t$-test was performed to examine the group differences regarding their perceptions of the external environment. The results demonstrated that the flow state had an overwhelming impact on a gamer's perceptions of their external environment $(t=2.14, \mathrm{df}=22, p<.05)$. The flow group $(\mathrm{M}=1.50, \mathrm{SD}=1.17)$ had significantly lower scores on the song recognition test when compared with the nonflow group $(\mathrm{M}=2.58, \mathrm{SD}=1.31)$, suggesting a decreased amount of perception of external information. Thus, (H3) was supported.

4.3. The Effect of Different Flow States on Gamer's Blink Rate. The ANOVA was performed on the gamer's blink rate with group (flow and nonflow) as between subjects factors and blink rate measurements as within subjects factors. The results showed that there was a significant difference between the flow group and the nonflow group on blink rate during the first 30 minutes of the game and when the boss appeared (at 60-70 minutes). The flow group had a significantly lower blink rate during the first ten minutes $(p<.05), 10-20 \mathrm{mi}-$ nutes into the game $(p<.05), 20-30$ minutes into the game $(p<.05)$, and when the boss appeared at 60-70 minutes ( $p=$ 0.07 ), compared to before the participants were immersed in the flow experience (see Figure 5). However, the nonflow group demonstrated no significant differences in blink rate over that time (see Figure 6). These results confirmed (H4).

\section{General Discussion}

The present study examined how two important personalities (dominance and compliance) affected people's flow experience and the external expressions of different flow 


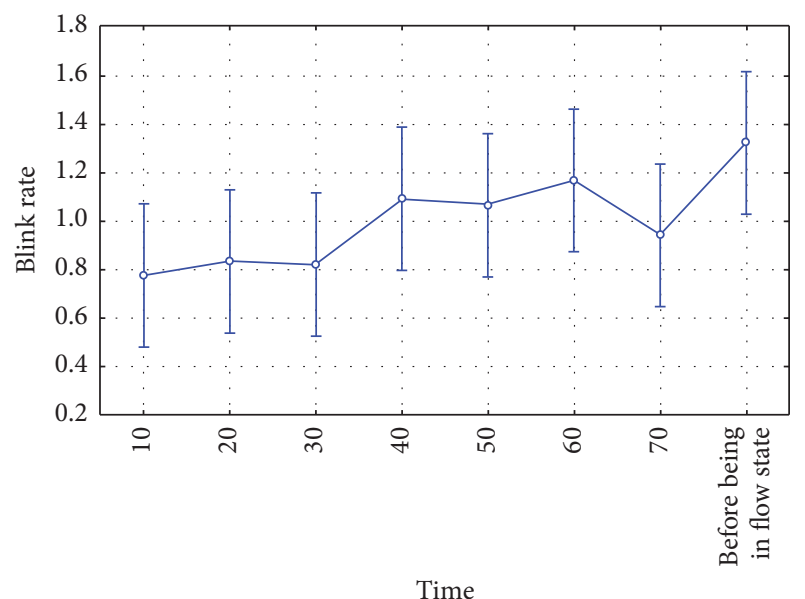

FIGURE 5: Blink rate result of flow group.

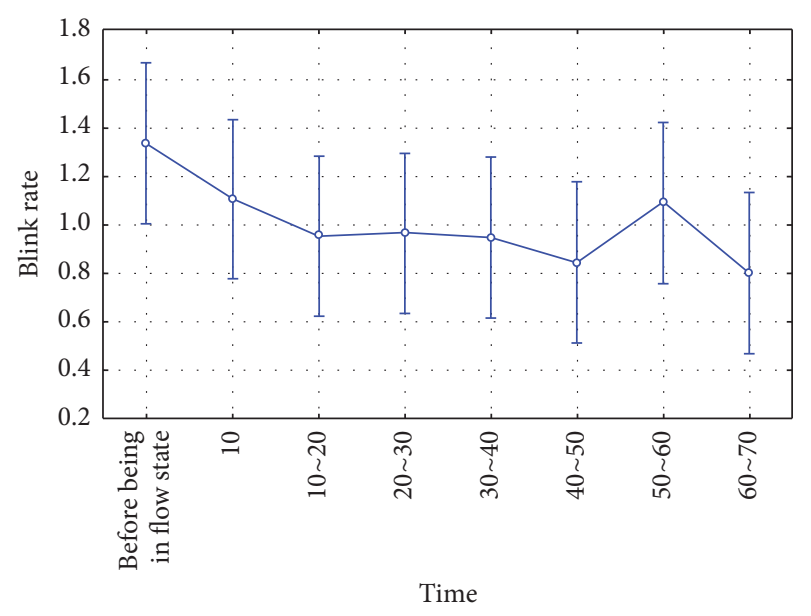

FIGURE 6: Blink rate result of nonflow group.

states. The results of our study confirmed all four hypotheses. People with dominant or noncompliant personalities experienced a much easier flow state compared to nondominant or compliant people. In addition, people in the flow state showed a decreased amount of perception of the external environment information, compared to people in the nonflow state. The most important result identified during the course of the study was that a reduced eye blink rate was found in the flow state, but this effect only appeared at the start of the game.

The results showed that dominant or noncompliant people were more immersed in the game than nondominant or compliant people, which was consistent with Mihaly's (1990) idea that the flow experience involves individual differences [7]. Mihaly found that some individuals relied upon their external environment and could not easily control their thinking. These people also could not easily enter into a flow status. The researcher suggested that self-perception could significantly affect flow. If one continuously worries about their surrounding environment, he/she will be afraid of making mistakes or creating a bad impression. As such, they are less likely to experience pleasure. Nevertheless, pleasure or playfulness is some of the key elements for experiencing flow, an idea that was supported by a study conducted by Mauri et al. (2011) [30]. Mauri et al. found that a specific positive affective state experienced by users might be one of the key elements in the success of Facebook [30]. In the present study, dominant or noncompliant gamers always do things they want to do and their behaviors are less likely to be restricted by the group. As such, they are likely to enjoy the game much more. However, for nondominant or compliant gamers, who may be more likely to accept the tasks or directions passively experienced a higher level of group restriction on their behaviors, it was more difficult to enjoy the game. These gamers had a lower probability of experiencing flow.

The results demonstrated that people in a flow state had a decreased amount of perception of their external environment information, which conformed to our expectations. More importantly, the present study found that the eye blink rate also decreased when people were in the flow state. This finding was in accordance with previous studies [25], which revealed that the eye blink rate dropped when the task required strong visual attention. The findings were also consistent with flow theory, which considered that a loss of self-consciousness and action awareness were fundamental dimensions that described the mindset in a flow state [20]. This phenomenon might account for the attention filter theory [21], which suggests that attention selects certain pieces of incoming sensory information for conscious processing and filters out the remaining sensory information from conscious processing. In this study, participants who entered the flow experience might filter out information from the external environment, such as the song played on the computer. The reduction of eye blink frequency in the flow state elicited by computer games may be associated with the cortical impact on the pontine reticular formation neurons, which is considered to induce spontaneous blinking [31]. The suppressed input from peripheral afferents may partly explain this effect [25]. Therefore, the results of the present study may indicate that eye blink rate is an objective indicator of flow experience.

The present study contributed to the expanding theoretical base of understanding the elusive online flow, having implications for game designers and service providers. First, immersion is one of the main motivations for users to play online game [32]. People with dominant and noncompliant personalities more easily experience flow, so this finding indicates that game designers should develop more characters that gamers can easily control. This will maintain the gamers' optimal status, flow. Second, the study found that eye blink rates and perception tasks in the external environment could be objective indicators of flow experience. This provides scholars and service providers with objective and effective tools to investigate users' behaviors.

However, there are also some limitations in the present study. This research was an explorative study with the small sample size not large enough to represent all online gamers. Therefore, the conclusions derived from this study should be treated cautiously. Future studies should use a larger sample size to confirm the findings identified during the 
course of this study. In addition, the present study could not identify specific mechanisms of how personality influenced flow experience. Further research is needed to explore the underlying mechanisms.

\section{Conclusions}

The present study found that personality (dominance and compliance) had a significant impact on flow experience. People with dominant or noncompliant personalities experienced more flow than participants with nondominant or compliant personalities. More importantly, the present study found a decreased perception of the external environment and a decreased blink rate in the flow state compared to in the nonflow state. This suggests that perceptions of the external environment and blink rate could be objective indicators of flow experience.

\section{Conflicts of Interest}

The authors declare that they have no conflicts of interest.

\section{Acknowledgments}

This study was funded by a National Natural Science Foundation of China Grant 71661167006.

\section{References}

[1] M. Csikszentmihalyi, Beyond Boredom and Anxiety, JosseyBass, San Francisco, Calif, USA, 1975.

[2] T. P. Novak and D. L. Hoffman, "Measuring the flow experience among web users," Interval Research Corporation, vol. 31, pp. 136, 1997.

[3] P. H. Lindsay and D. A. Norman, Human Information Processing: An Introduction to Psychology, Academic Press, NY, USA, 1972.

[4] J. J. Fins and N. D. Schiff, In the Blink of the Mind's Eye, WileyBlackwell, Hoboken, NJ, USA, 2010.

[5] A. W. Lukaszewski and J. R. Roney, "Estimated hormones predict women's mate preferences for dominant personality traits," Personality and Individual Differences, vol. 47, no. 3, pp. 191-196, 2009.

[6] M. Csikszentmihalyi and J. LeFevre, "Optimal experience in work and leisure," Journal of Personality and Social Psychology, vol. 56, no. 5, pp. 815-822, 1989.

[7] C. Mihaly, Flow: The Psychology of Optimal Experience, Harper and Row, New York, NY, USA, 1990.

[8] S. H. Han and K. M. Yoo, "A study on the effects of autotelic personality on flow experience: focus on the bicycle riders," Journal of Tourism Studies, vol. 26, no. 5, pp. 45-72, 2014.

[9] K. Heller, C. Bullerjahn, and R. Von Georgi, "The relationship between personality traits, flow-experience, and different aspects of practice behavior of amateur vocal students," Frontiers in Psychology, vol. 6, Article ID 01901, 2015.

[10] J. Keller and F. Blomann, "Locus of control and the flow experience: an experimental analysis," European Journal of Personality, vol. 22, no. 7, pp. 589-607, 2008.

[11] D. G. Myers, Social Psychology, McGraw-Hill Education, New York, NY, USA, 1987.
[12] S. A. Hackley, M. Á. Muñoz, K. Hebert, F. Valle-Inclán, and J. Vila, "Reciprocal modulation of eye-blink and pinna-flexion components of startle during reward anticipation," Psychophysiology, vol. 46, no. 6, pp. 1154-1159, 2009.

[13] S. R. Ross and H. N. Keiser, "Autotelic personality through a five-factor lens: Individual differences in flow-propensity," Personality and Individual Differences, vol. 59, pp. 3-8, 2014.

[14] C. Boersch and F. von Diest, Flow: Das Geheimnis des Glücks, Stuttgart: Klett-Cotta, Germany, 2010.

[15] M. Csikszentmihalyi and J. Nakamura, "Effortless attention in everyday life: a systematic phenomenology," in Effortless Attention: A New Perspective in the Cognitive Science of Attention and Action, B. Bruya, Ed., pp. 179-190, The MIT Press, Cambridge, UK, 2010.

[16] E. S. Reed, "Perception is to self as memory is to selves," in The Remembering Self Construction And Accuracy in The SelfNarrative, U. Neisser and R. Fivush, Eds., vol. 15, pp. 278-292, Cambridge University Press, Oxford, UK, 1994.

[17] S. Kühn, R. Lorenz, T. Banaschewski et al., "Positive association of video game playing with left frontal cortical thickness in adolescents," PLoS ONE, vol. 9, no. 3, Article ID e91506, 2014.

[18] S. Yan and M. S. El-Nasr, "Visual attention in 3D video games," in Proceedings of the Symposium on Eye Tracking Research and Applications (ETRA'06), p. 42, San Diego, Calif, USA, March 2006.

[19] D. L. King, M. Gradisar, A. Drummond et al., "The impact of prolonged violent video-gaming on adolescent sleep: An experimental study," Journal of Sleep Research, vol. 22, no. 2, pp. 137-143, 2013.

[20] T. Strandvall, "Eye tracking in human-computer interaction and usability research," Lecture Notes in Computer Science (including subseries Lecture Notes in Artificial Intelligence and Lecture Notes in Bioinformatics), vol. 5727, no. 2, pp. 936-937, 2009.

[21] G. Devetag, S. Di Guida, and L. Polonio, "An eye-tracking study of feature-based choice in one-shot games," Experimental Economics, vol. 19, no. 1, pp. 177-201, 2016.

[22] A. Kenny, H. Koesling, D. Delaney, S. McLoone, and T. Ward, "A preliminary investigation into eye gaze data in a first person shooter game," in Proceedings of the 19th European Conference on Modelling and Simulation (ECMS'05), pp. 733-740, Riga, Latvia, June 2005.

[23] S. Alkan and K. Cagiltay, "Studying computer game learning experience through eye tracking: Colloquium," British Journal of Educational Technology, vol. 38, no. 3, pp. 538-542, 2007.

[24] J. A. Veltman and A. W. K. Gaillard, "Physiological workload reactions to increasing levels of task difficulty," Ergonomics, vol. 41, no. 5, pp. 656-669, 1998.

[25] M. C. Acosta, J. Gallar, and C. Belmonte, "The influence of eye solutions on blinking and ocular comfort at rest and during work at video display terminals," Experimental Eye Research, vol. 68, no. 6, pp. 663-669, 1999.

[26] Z. W. Wu, C. Begley, J. Zhang, L. A. Adebisi, and T. Simpson, "Does ocular surface stimulation oppose internal controls over blinking?" ARVO Annual Meeting Abstract Search and Program Planner, p. 3846, 2011.

[27] K. Iwanaga, S. Saito, Y. Shimomura, H. Harada, and T. Katsuura, "The effect of mental loads on muscle tension, blood pressure and blink rate," Journal of Physiological Anthropology and Applied Human Science, vol. 19, no. 3, pp. 135-141, 2000.

[28] J. Y. Kim, S. N. Min, and Y. D. Park, "Evaluation of flow experience by using psychophysiological visual feedbacks," Journal 
of the Ergonomics Society of Korea, vol. 32, no. 6, pp. 481-487, 2013.

[29] T. J. Chou and C. C. Ting, "The role of flow experience in cybergame addiction," CyberPsychology \& Behavior, vol. 6, no. 6, pp. 663-675, 2003.

[30] M. Mauri, P. Cipresso, A. Balgera, M. Villamira, and G. Riva, "Why is Facebook so successful? Psychophysiological measures describe a core flow state while using Facebook," Cyberpsychology, Behavior, and Social Networking, vol. 14, no. 12, pp. 723-731, 2011.

[31] C. N. Karson, "Blinking," Bulletin of the Social Belge Ophthalmology, vol. 237, pp. 443-457, 1989.

[32] N. Yee, "Motivations for play in online games," CyberPsychology \& Behavior, vol. 9, no. 6, pp. 772-775, 2006. 

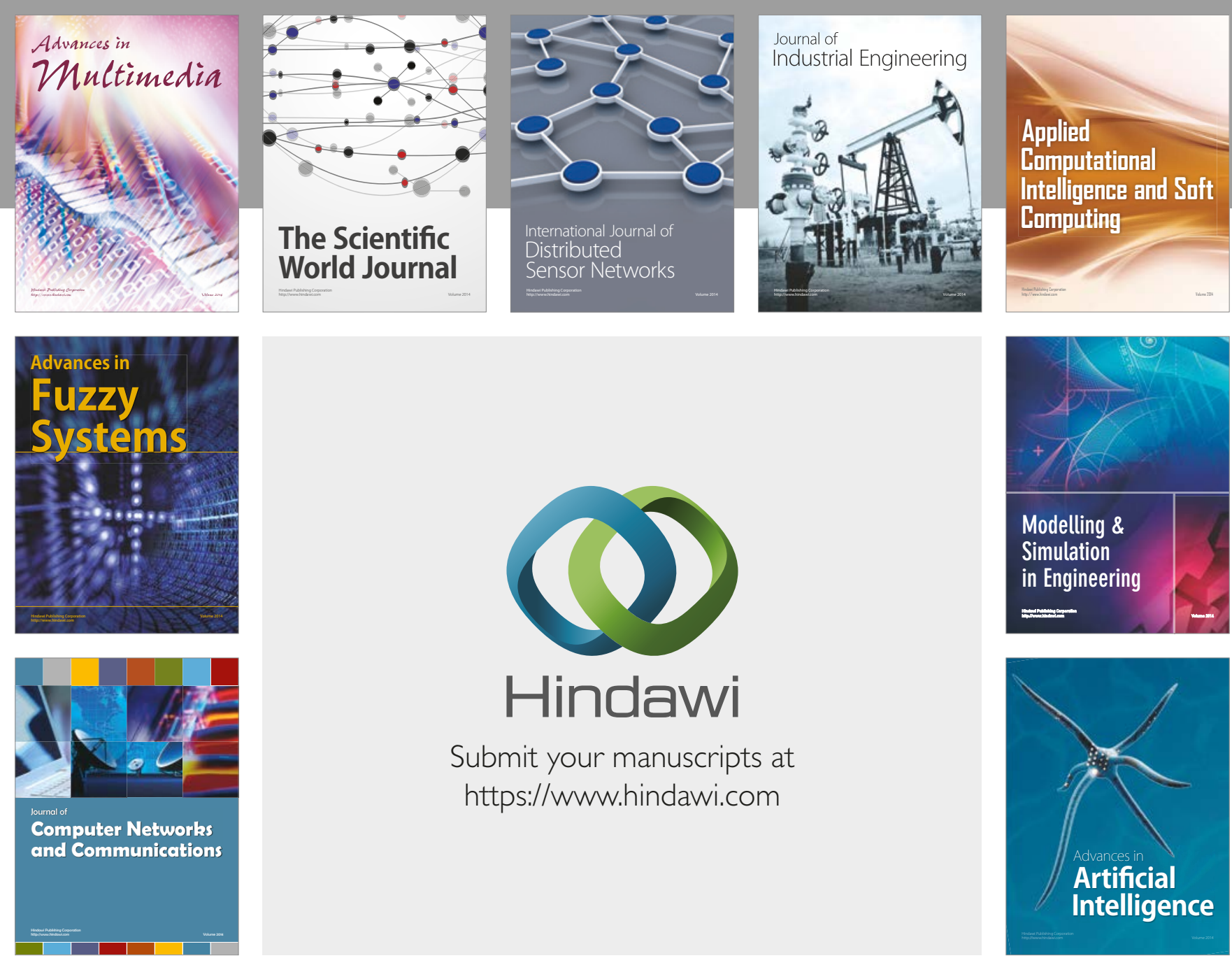

\section{Hindawi}

Submit your manuscripts at

https://www.hindawi.com
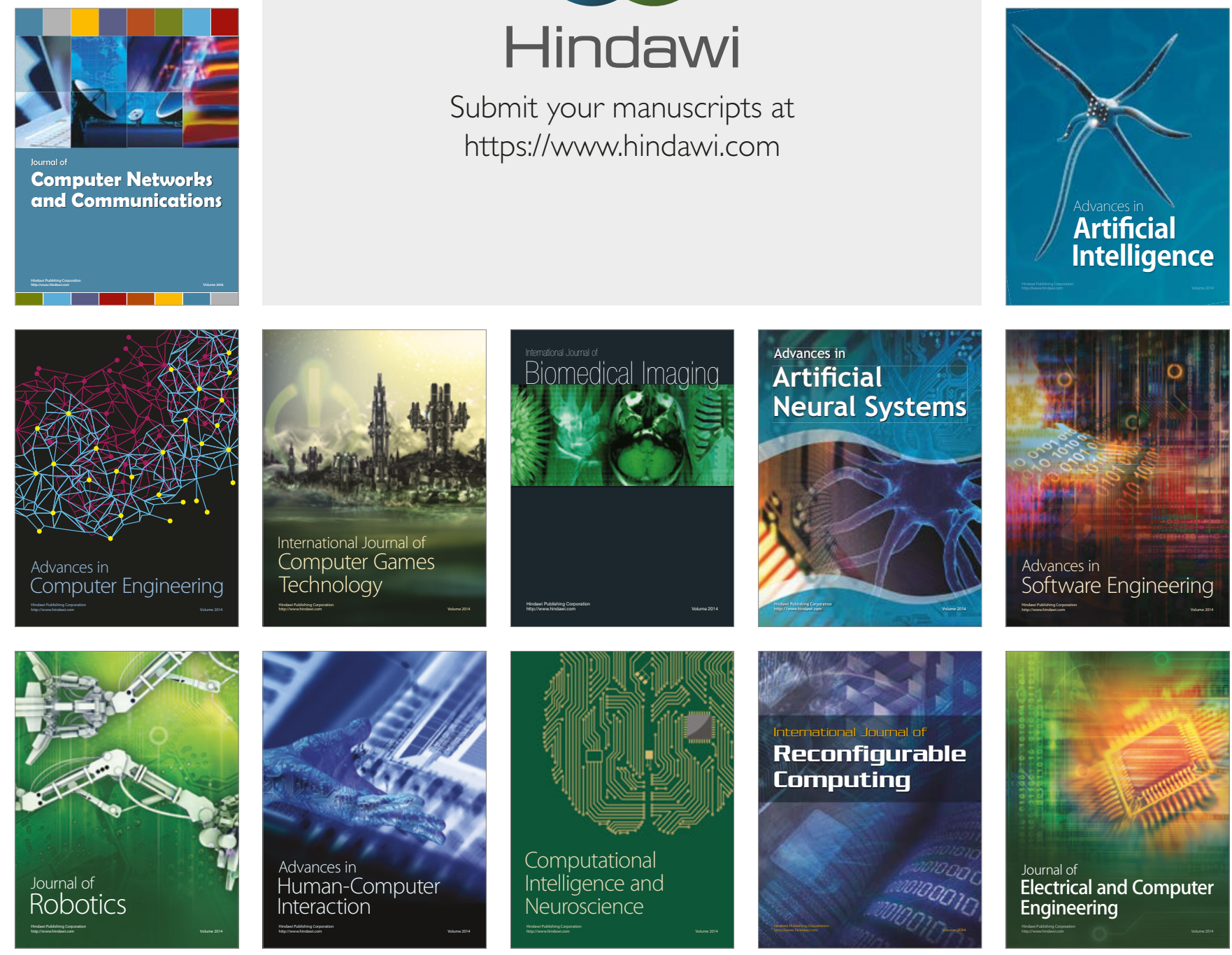